\title{
Assessment of four serological techniques in the immunological diagnosis of farmers' lung disease
}

Correspondence

Gabriel Reboux

gabriel.reboux@univ-fcomte.fr

Received 18 September 2006

Accepted 29 May 2007

\section{Gabriel Reboux, ${ }^{1}$ Renaud Piarroux, ${ }^{1}$ Sandrine Roussel, ${ }^{1}$ Laurence Millon, Karine Bardonnet ${ }^{1}$ and Jean-Charles Dalphin ${ }^{2}$}

\author{
${ }^{1}$ Department of Mycology, University Hospital, Besançon, France \\ ${ }^{2}$ Department of Respiratory Disease, University Hospital, Besançon, France
}

\begin{abstract}
Farmers' lung disease (FLD) is a pulmonary disease that results from repeated inhalation of antigens from mouldy hay or straw. The objective of this prospective study was to assess the reliability of four serological techniques in FLD diagnosis. Sera from 15 consecutive patients with FLD, 15 healthy control farmers and 30 urban controls were analysed using four serological techniques [electrosyneresis (ES), Ouchterlony double diffusion (DD), ELISA and Western blot (WB)] with four antigens (Absidia corymbifera, Eurotium amstelodami, Wallemia sebi and Saccharopolyspora rectivirgula). In the authors' region, ES on cellulose acetate with $A$. corymbifera antigen was the most relevant diagnostic tool for discriminating FLD patients from healthy exposed farmers (sensitivity $87 \%$, specificity $100 \%$ ). DD tests were in accordance with $\mathrm{ES}$, but their discriminatory power was lower. No threshold indicating both good sensitivity and specificity could be established with ELISA. WB analysis failed to identify specific bands for FLD. This study demonstrates the efficacy of determining precipitin levels with an appropriate technique, using a panel of antigens consistent with the specific exposure of a given area.
\end{abstract}

\section{INTRODUCTION}

Farmers' lung disease (FLD), the most common form of extrinsic allergic alveolitis, is a pulmonary disease that results from repeated exposure to an inhalation of antigens from mouldy hay or straw, which leads to sensitization and development of granulomatous lung reactions. Its diagnosis has most often relied on an array of non-specific clinical symptoms and signs developed in an appropriate setting, with the demonstration of interstitial marking on chest X-rays, lymphocytic alveolitis on bronchoalveolar lavage, serum precipitins against offending antigens and/or a granulomatous reaction on lung biopsies (American Thoracic Society, 1998; Patel et al., 2001).

The diagnostic value of serological tests in FLD is controversial (Burrell \& Rylander, 1981; Dalphin et al., 1994). For some authors, serology is a good diagnostic tool, whereas for others, it fails to discriminate FLD subjects from healthy exposed subjects and appears to be a mere indicator of exposure (American Thoracic Society, 1998; Cormier \& Belanger, 1989; Cormier et al., 2004; Lacasse et al., 2003). This discrepancy may be due to the choice of antigens and/or the serological techniques applied, or to

Abbreviations: DD, Ouchterlony double diffusion; ES, electrosyneresis; FLD, farmers' lung disease; ROC, receiver-operating characteristic; WB, Western blot.

Serological techniques are described completely in supplementary data available with the online version of this paper. the methodology used in studies which focus on diagnostic criteria for cases and characteristics and level of exposure for controls.

In Franche-Comté, a region in the east of France where FLD is common, a previous study demonstrated the poor reliability of immunological tests using Saccharopolyspora rectivirgula despite the clinical probability of FLD being correlated with the presence of precipitins against total hay extracts (Dalphin et al., 1994). Another study demonstrated that Absidia corymbifera, Eurotium amstelodami and, to a lesser degree, Wallemia sebi were probably the main causes of FLD in eastern France, whereas $S$. rectivirgula might not play a major role in our region (Reboux et al., 2001), as is probably the case in other countries (Erkinjuntti-Pekkanen et al., 1999).

The aim of this study was to assess the diagnostic value of four serological techniques for FLD: electrosyneresis (ES), Ouchterlony double diffusion (DD), ELISA and Western blot (WB) analysis. We used the three antigens specifically involved in FLD in the east of France and S. rectivirgula, the classic FLD antigen. Three groups of subjects were compared: FLD patients, and exposed and unexposed controls.

\section{METHODS}

Subjects. In this prospective case-control study, immunological reactions were compared in FLD dairy farmers $(n=15)$, control dairy 
farmers $(n=15)$ and non-exposed urban subjects $(n=30)$. FLD patients were consecutive cases diagnosed according to the latest published criteria (Schuyler \& Cormier, 1997). Each patient was asked to designate a control subject comparable to him/herself with respect to the classic criteria (age, gender and smoking habits) and professional activity (size, type and geographical location of the farm, structure of the cattle shed, mode of drying and storing fodder, and daily duration of exposure). A standardized questionnaire and auscultation were used to ensure that none of the control subjects presented any general or respiratory symptoms. Thirty non-exposed urban controls were recruited in the same region (towns up to 5000 residents) and matched to the case subjects for age ( \pm 5 years), smoking habits and gender. Informed written consent was obtained from each subject. The protocol was approved by the local review board for research involving human subjects.

\section{Immunological methods}

Antigen extract. Three somatic antigens were derived from fungi isolated from hay from the Franche-Comté region and referenced by the BCCM/IHEM collection, Brussels, Belgium [A. corymbifera (IHEM 16288), E. amstelodami (IHEM 16286) and W. sebi (IHEM 16284), and one somatic antigen was derived from the reference Lacey's strain S. rectivirgula (ATCC 15347)]. The antigens were produced as previously described (Reboux et al., 2001). Briefly, both fungi and bacteria strains were cultured in brain heart infusion for 2 weeks, crushed by Ultraturax (IKA Labortechnik), sonicated, extracted overnight in $\mathrm{NH}_{4} \mathrm{CO}_{3}$ at $4{ }^{\circ} \mathrm{C}$, centrifuged at 13000 r.p.m., ultrafiltered on Centricon 10 (Amicon Millipore) and dosed for standardization at $100 \mathrm{mg}$ protein $\mathrm{ml}^{-1}$.

Serology techniques. All sera were tested by four techniques at the same time and with the same batches of antigen. Serum precipitins were investigated by agar gel DD (Ouchterlony, 1953) and ES on cellulose acetate (Gari et al., 1982). All results were read blindly by two operators; no difference was noted between the two readings. IgG and IgA levels were investigated by ELISA with peroxidase-conjugated anti-human IgG and IgA (Engvall \& Perlman, 1971). WB analysis was done with alkaline phosphatase-conjugated anti-human IgG and IgA (Laemmli, 1970). Serological techniques are described completely in supplementary data published in the online version of the journal.

Statistical analyses. Results from FLD patients and farmer controls were evaluated by the receiver-operating characteristic (ROC) (Zweig \& Campbell, 1993). Sensitivity and specificity tables (MedCalc Software v7.2; Mariakerke, Belgium) were used to determine the optimum cut-off level. A non-parametric Wilcoxon signed ranked test was used to compare the number of bands obtained with the WB technique. $P$ values less than 0.05 were considered significant.

\section{RESULTS AND DISCUSSION}

The assessment of immunodiffusion methods and ELISA to discriminate FLD patients from farmer controls $(n=15)$ is presented in Tables 1 and 2. In our region, ES on cellulose acetate with the A. corymbifera antigen showed the highest sensitivity and specificity $(87 \%$ and $100 \%$, respectively) when a two-arc threshold was selected by ROC curve analysis. The ES and DD techniques actually appeared to be more specific than ELISA and WB in diagnosing FLD.

ES showed the best discriminatory power, even though results varied depending on the antigens used (Table 1). A range of $0-8$ arcs of precipitins was observed in FLD patients. A two-arc threshold differentiated 13 of the 15 cases from control subjects, with no false-positive results, with the A. corymbifera antigen and only 7 out of 15 cases with the W. sebi antigen (Fig. 1). A three-arc threshold differentiated 10 out of 15 cases with the E. amstelodami antigen and only 5 out of 15 cases, including one falsepositive result, with the $S$. rectivirgula antigen. For all antigens, non-exposed urban subjects consistently had a far lower number of arcs (maximum of one for $9 \%$ of all sera tested) than farmers without symptoms.

DD gave comparable results, but the number of arcs of precipitins was lower (0-3 arcs) (Table 1$)$. Notably, a onearc threshold with the A. corymbifera antigen detected 10 out of 15 cases, but also led to one false-positive result. One arc was obtained for $10 \%$ of urban controls with each antigen except $S$. rectivirgula.

Using these thresholds defined by ROC curve analysis, ELISA IgG was positive with the A. corymbifera antigen in 8 out of 15 FLD patients (2 false positives) (Fig. 2). The IgG test using the three other antigens was positive in fewer

Table 1. Sensitivity and specificity of serological tests (electrosyneresis and double diffusion) with the four antigens

\begin{tabular}{|c|c|c|c|c|c|c|}
\hline Antigen & $\begin{array}{l}\text { Area under the ROC } \\
\text { curve (SD) }\end{array}$ & $\begin{array}{l}\text { Selected } \\
\text { threshold }\end{array}$ & $\begin{array}{l}\text { Sensitivity } \\
(95 \% \mathrm{CI})\end{array}$ & $\begin{array}{l}\text { Specificity } \\
(95 \% \mathrm{CI})\end{array}$ & $\mathbf{L R}+{ }^{*}$ & $\begin{array}{l}\text { Odds ratio } \\
(\text { CI } 95 \%)\end{array}$ \\
\hline \multicolumn{7}{|l|}{ Electrosyneresis } \\
\hline A. corymbifera & $0.944(0.045)$ & $2 \operatorname{arcs}$ & $86.7(59.5-98.0)$ & $100(100-100)$ & - & - \\
\hline E. amstelodami & $0.871(0.067)$ & $3 \operatorname{arcs}$ & $66.7(38.4-88.1)$ & $100(100-100)$ & - & - \\
\hline W. sebi & $0.687(0.098)$ & $2 \operatorname{arcs}$ & $46.7(21.3-73.4)$ & $93.3(68.0-98.9)$ & 7.00 & $12.25(1.69-88.94)$ \\
\hline S. rectivirgula & $0.756(0.090)$ & $2 \operatorname{arcs}$ & $46.7(21.3-73.4)$ & $93.3(68.0-98.9)$ & 7.00 & $5.69(1.03-31.46)$ \\
\hline \multicolumn{7}{|l|}{ Double diffusion } \\
\hline A. corymbifera & $0.813(0.080)$ & $1 \operatorname{arc}$ & $73.3(44.9-92.0)$ & $93.3(68.0-98.9)$ & 11.00 & $38.5(5.64-262.60)$ \\
\hline E. amstelodami & $0.713(0.095)$ & 1 arc & $53.3(26.6-78.7)$ & $86.7(59.5-98.0)$ & 4.00 & $7.43(1.37-40.32)$ \\
\hline$W$. sebi & $0.687(0.098)$ & 1 arc & $73.3(44.9-92.0)$ & $60.0(32.3-83.6)$ & 1.83 & $4.12(0.91-18.63)$ \\
\hline S. rectivirgula & $0.698(0.097)$ & $1 \operatorname{arc}$ & $66.7(38.4-88.1)$ & $66.7(38.4-88.1)$ & 2.00 & $4(0.9-17.72)$ \\
\hline
\end{tabular}

${ }^{\star} \mathrm{LR}+$, Positive likelihood ratio. 
Table 2. Sensitivity and specificity of serological tests (ELISA $\lg G$ and $\lg A$ ) with the four antigens

\begin{tabular}{|c|c|c|c|c|c|c|}
\hline Antigen & $\begin{array}{c}\text { Area under the ROC } \\
\text { curve (SD) }\end{array}$ & $\begin{array}{l}\text { Selected } \\
\text { threshold }\end{array}$ & $\begin{array}{l}\text { Sensitivity } \\
(95 \% \mathrm{CI})\end{array}$ & $\begin{array}{l}\text { Specificity } \\
(95 \% \mathrm{CI})\end{array}$ & $\mathrm{LR}+{ }^{*}$ & $\begin{array}{l}\text { Odds ratio } \\
\text { (CI } 95 \%)\end{array}$ \\
\hline \multicolumn{7}{|l|}{ ELISA IgG $\dagger$} \\
\hline E. amstelodami & $0.620(0.103)$ & $>534 \dagger$ & $46.7(21.3-73.4)$ & $86.7(59.5-98.0)$ & 3.50 & $3.79(0.79-18.26)$ \\
\hline W. sebi & $0.758(0.089)$ & $>195 \dagger$ & $60.0(32.3-83.6)$ & $93.3(68.0-98.8)$ & 9.00 & $21(3.06-144.09)$ \\
\hline S. rectivirgula & $0.624(0.103)$ & $>153 \dagger$ & $86.7(59.5-98.0)$ & $40.0(16.4-67.7)$ & 1.44 & $4.33(0.76-24.70)$ \\
\hline A. corymbifera & $0.638(0.102)$ & $\leqslant 104 \dagger$ & $53.3(26.6-78.7)$ & $80.0(51.9-95.4)$ & 2.67 & $4.57(0.95-22.03)$ \\
\hline E. amstelodami & $0.644(0.100)$ & $\leqslant 19 \dagger$ & $33.3(11.9-61.6)$ & $93.3(68.0-98.9)$ & 5.00 & $7(0.87-56.54)$ \\
\hline W. sebi & $0.522(0.107)$ & $>256 \dagger$ & $40.0(16.4-67.7)$ & $86.7(59.5-98.0)$ & 3.00 & $4.33(0.76-24.70)$ \\
\hline S. rectivirgula & $0.547(0.107)$ & $\leqslant 44 \dagger$ & $33.3(11.9-61.6)$ & $93.3(68.0-98.9)$ & 5.00 & $7(0.87-56.54)$ \\
\hline
\end{tabular}

${ }^{\star} \mathrm{LR}+$, Positive likelihood ratio.

$\dagger$ Absorbance.

than 7 out of 15 FLD patients. Although the level of IgG antibodies was often higher in patients than in controls, no threshold indicating both good sensitivity and specificity could be established. Moreover, results for $13 \%$ of cases and $12 \%$ of farmer controls were below those of the pooled urban control sera for all four antigens. Surprisingly, the ELISA IgA test used with A. corymbifera, E. amstelodami and $S$. rectivirgula antigens showed the highest levels of antibodies in sera from seven control farmers and lower levels in five FLD sera than in pooled urban sera.

WB IgG and IgA analysis failed to identify specific bands for FLD. The number of bands revealed with the IgG conjugate in the FLD group varied from 6 to 18 bands for A. corymbifera (see Supplementary Fig. S2 in the online version of the journal), from 6 to 16 for E. amstelodami, from 5 to 21 for $W$. sebi and from 10 to 24 for $S$. rectivirgula. There were fewer bands with the IgA conjugate than with IgG. Neither conjugate revealed a significant difference in the number of bands between FLD patients and farmer controls. Some urban controls presented with positive IgG WB results, with a maximum number of bands of 11, 5, 5 and 9 for A. corymbifera, E. amstelodami, $W$. sebi and $S$. rectivirgula antigens, respectively.

Finally, ES and DD techniques appeared to be more specific than ELISA and WB analysis in diagnosing FLD. The presence of precipitins has previously been reported to indicate exposure to micro-organisms only (Cormier \& Belanger, 1989; Cormier et al., 2004; Gariepy et al., 1989; Katila \& Mäntyjärvi, 1987). The sensitivity of DD varies considerably, ranging from 6 to $100 \%$ (Gruchow et al., 1981; Ojanen, 1992; Pépys, 1969). Obviously, the results depend on the balance between the antigens used for serological diagnosis and the micro-organisms actually involved in the disease in a given area. Consequently, several authors have recommended adapting a panel of antigens specific to each area in order to improve the value

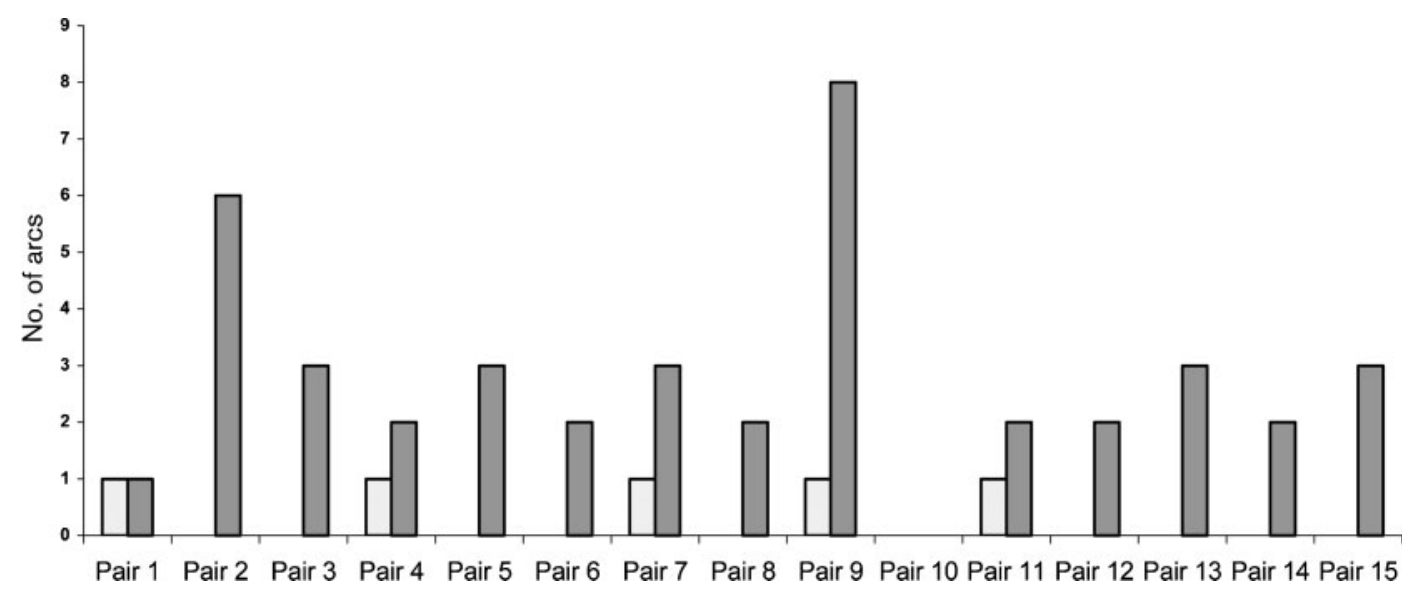

Fig. 1. Results comparing the number of arcs by ES with the $A$. corymbifera antigen in exposed controls (left side of each pair) and FLD cases (right side of each pair). 


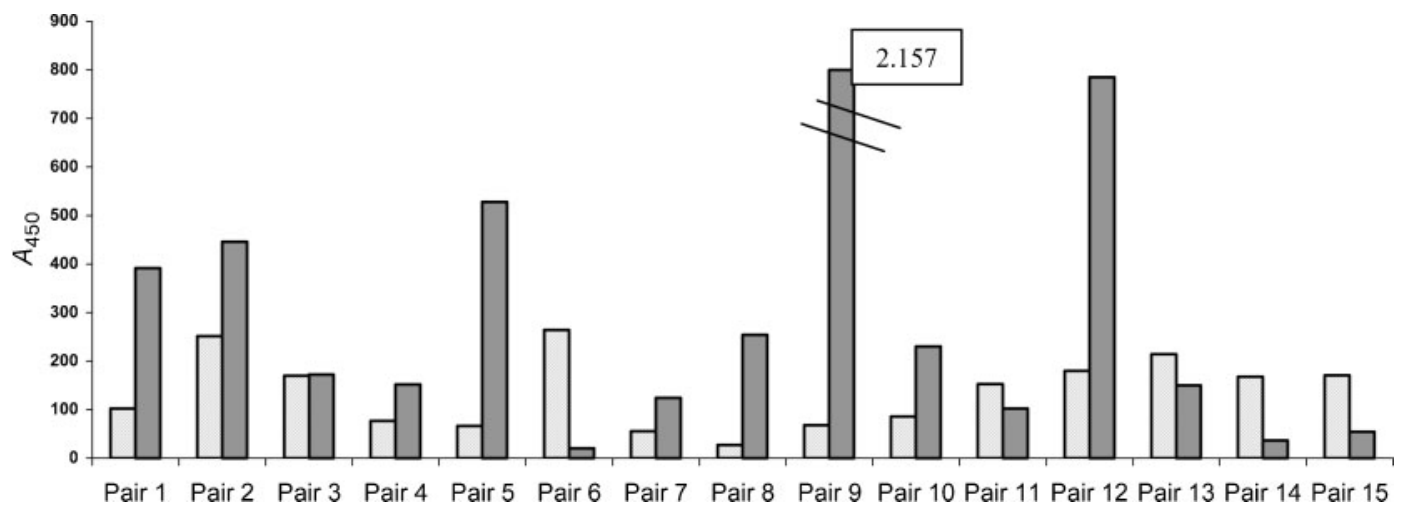

Fig. 2. Results comparing the absorbance by ELISA IgG with the $A$. corymbifera antigen in exposed controls (left side of each pair) and FLD cases (right side of each pair).

of diagnostic tests (Katila \& Mäntyjärvi, 1987; Patel et al., 2001). Our study strongly supports this recommendation. ES on cellulose acetate is based on the same principle as DD, but antigen-antibody migration is activated by an electric current in an electrophoresis chamber. In our study, this technique, which can be done in $4 \mathrm{~h}$ and is simple enough to be used for routine diagnosis, led to diagnosis of FLD in $87 \%$ of cases with A. corymbifera antigen, with no false-positive results.

Some authors have reported that immunoglobulin levels measured using ELISA assays are only indicators of exposure to the corresponding micro-organisms (Lappalainen et al., 1998; Rautalahti et al., 1990). Others have shown immunological profiles grouping several antigens and isotypes (Brummund et al., 1988; Ojanen, 1992) to distinguish between FLD and exposed controls. In our study, it was not possible to distinguish FLD cases from controls with both good specificity and good sensitivity for any of the four antigens tested, either for IgG or for IgA antibodies. Using WB analysis, we did not find specific bands or patterns which could help to diagnose FLD patients. The reason for the discrepancy between techniques revealing precipitins and those revealing IgG or IgA remains unclear. The 'massive' precipitin reaction obtained with FLD subjects can be explained by the reaction of antibodies to antigens with a high molecular mass. These antibodies seem more relevant for FLD diagnosis than those detected by WB analysis, which reacted with multiple lightweight antigens $(100-10 \mathrm{kDa})$. Hence a better diagnostic value was demonstrated for ES, probably due to a better separation of high-molecular-mass antigens by electric current.

In conclusion, our study demonstrates the dual necessity of using a panel of antigens consistent with the specific exposure of a given area in conjunction with the appropriate serological technique. Our results suggest that ES on cellulose acetate with a panel of antigens including $A$. corymbifera is a relevant diagnostic tool which can discriminate FLD patients from healthy exposed farmers.

\section{ACKNOWLEDGEMENTS}

The authors are grateful to Nancy Richardson-Peuteuil for her editorial assistance. This work was supported by EA 2276, 'Santé et Environnement Rural, Université de Franche-Comté', France (SERF).

\section{REFERENCES}

American Thoracic Society (1998). Respiratory health hazards in agriculture. Medical Section of the American Lung Association. Am J Respir Crit Care Med 158, S1-S76.

Brummund, W., Kurup, V. P., Resnick, A., Milson, T. J. \& Fink, J. N. (1988). Immunologic response to Faenia rectivirgula (Micropolyspora faeni) in a dairy family. J Allergy Clin Immunol 82, 190-195.

Burrell, P. \& Rylander, R. (1981). A critical review of the role of precipitins in hypersensitivity pneumonitis. Eur J Respir Dis 62, 332-343.

Cormier, Y. \& Belanger, J. (1989). The fluctuant nature of precipitating antibodies in dairy farmers. Thorax 44, 469-473.

Cormier, Y., Letourneau, L. \& Racine, G. (2004). Significance of precipitins and asymptomatic lymphocytic alveolitis: a 20 -yr followup. Eur Respir J 23, 523-525.

Dalphin, J. C., Toson, B., Monnet, E., Pernet, D., Dubiez, A., Laplante, J. J., Aiache, J. M. \& Depierre, A. (1994). Farmer's lung precipitins in Doubs (a department of France): prevalence and diagnostic value. Allergy 49, 744-750.

Engvall, E. \& Perlman, P. (1971). Enzyme-linked immunosorbent assay (ELISA). Quantitative assay of immunoglobulin G. Immunochemistry 8, 871-874.

Erkinjuntti-Pekkanen, R., Reiman, M., Kokkarinen, J. I., Tukiainen, H. O. \& Terho, E. O. (1999). IgG antibodies, chronic bronchitis, and pulmonary function values in farmer's lung patients and matched controls. Allergy 54, 1181-1187.

Gari, M., Smets, P., Pinon, J. M., Recco, P. \& Seguela, J. P. (1982). Use of immuno-electro-diffusion on cellulose acetate for the research of the precipitating antibodies in the screening of patients suspected of farmer's lung disease. Mycopathologia 78, 151-153.

Gariepy, L., Cormier, Y., Laviolette, M. \& Tardif, A. (1989). Predictive value of bronchoalveolar lavage cells and serum precipitins in asymptomatic dairy farmers. Am Rev Respir Dis 140, 1386-1389. 
Gruchow, H. W., Hoffman, R. G., Marx, J. J., Jr, Emanuel, D. A. \& Rimm, A. A. (1981). Precipitating antibodies of farmer's lung antigens in a Wisconsin farming population. Am Rev Respir Dis 124, 411-415.

Katila, M. L. \& Mäntyjärvi, R. A. (1987). Prognosis value of precipitins for working ability in dairy farmers. Eur J Respir Dis Suppl 152, $146-154$

Lacasse, Y., Selman, M., Costabel, U., Dalphin, J. C., Ando, M., Morell, F., Erkinjuntti-Pekkanen, R., Müller, N., Colby, T. V. \& other authors (2003). Clinical diagnosis of hypersensitivity pneumonitis. Am J Respir Crit Care Med 168, 952-958.

Laemmli, U. K. (1970). Cleavage of structural proteins during the assembly of the head of bacteriophage T4. Nature 227, 680-685.

Lappalainen, S., Pasanen, A. L., Reiman, M. \& Kalliokoski, P. (1998). Serum IgG antibodies against Wallemia sebi and Fusarium species in Finnish farmers. Ann Allergy Asthma Immunol 81, 585-592.

Ojanen, T. (1992). Class specific antibodies in serodiagnosis of farmer's lung. Br J Ind Med 49, 332-336.
Ouchterlony, O. (1953). Antigen-antibody reaction in gels. Acta Pathol Microbiol Scand 32, 231-240.

Patel, A. M., Ryu, J. H. \& Reed, C. E. (2001). Hypersensitivity pneumonitis: current concepts and future questions. J Allergy Clin Immunol 108, 661-670.

Pépys, J. (1969). Hypersensitivity diseases of the lungs due to fungi and organic dusts. Monogr Allergy 4, 1-147.

Rautalahti, M., Terho, E. O. \& Ojanen, T. (1990). Changes in the titers of IgG antibodies against farmer's lung antigens in the sera of healthy dairy farmers. Ann Allergy 64, 455-458.

Reboux, G., Piarroux, R., Mauny, F., Madroszyk, A., Millon, L., Bardonnet, K. \& Dalphin, J. C. (2001). Role of molds in farmer's lung disease in Eastern France. Am J Respir Crit Care Med 163, 1534-1539.

Schuyler, M. \& Cormier, Y. (1997). The diagnosis of hypersensitivity pneumonitis. Chest 111, 534-536.

Zweig, M. H. \& Campbell, G. (1993). Receiver-operating characteristic (ROC) plots: a fundamental evaluation tool in clinical medicine. Clin Chem 39, 561-577. 